\title{
APPLICATION OF MUNICH AGILE CONCEPTS FOR MBSE AS A HOLISTIC AND SYSTEMATIC DESIGN OF URBAN AIR MOBILITY IN CASE OF DESIGN OF VERTIPORTS AND VERTISTOPS
}

\author{
Salehi, Vahid; \\ Wang, Shirui \\ Institut of Enigneering Design of Mechatronic Systems
}

\begin{abstract}
This paper will adopt the Munich Agile Concepts (MAGIC) on the design of Vertistops and Vertiports (Verti-X) for Urban Air Mobility (UAM) Systems. Vertiports and Vertistops are single or multilanding spaces for air taxis, which are electric-powered vertical take-off and landing (eVTOLs) aircraft. The target of this paper is to apply a holistic approach how to design so called Verti-X in a holistic and system of system method from the Requirement-, Function-. Architecture- and the Implementation phase. The MAGIC approach contains all of the requierd phases which are necessary to build up such a complex design of Verti-X. Therefore, based on the literature survey, we have determined the key components of the Verti-X design with a thorough requirement analysis. Then, we have followed the existed criteria and regulations to design a valid and verified Verti-X which fulfills all the requirement. Furthermore, the focus of this paper is to apply the first four phases of MAGIC regarding the requirement, function, architecture and the implementation of such Verti-X Design.
\end{abstract}

Keywords: Systems Engineering (SE), Conceptual design, Industrial design

Contact:

Salehi, Vahid

Munich University of Applied Sciences

Mechatronics

Germany

salehi-d@hm.edu

Cite this article: Salehi, V., Wang, S. (2021) 'Application of Munich Agile Concepts for MBSE as a Holistic and Systematic Design of Urban Air Mobility in Case of Design of Vertiports and Vertistops', in Proceedings of the International Conference on Engineering Design (ICED21), Gothenburg, Sweden, 16-20 August 2021. DOI:10.1017/ pds. 2021.50 


\section{INTRODUCTION}

Urban Air Mobility (UAM) is a secure and efficient urban air traffic system that covers a much wider area of passenger and cargo transport scenarios than the typical multi-rotor drone. Urban air traffic networks are undergoing electrification, smart and unmanned improvements, much like the automotive industry. Electric vertical take-off and landing (eVTOL) aircraft production, fully automated piloting, and air traffic management promise to revolutionize inter- and intra-city transport. Vertistops are the smallest type of UAM ground infrastructure in this scenario. Since it consists only of a runway and a maximum of two landing pads, it is planned to be built anywhere. Vertiports, on the other hand, are the intermediate category for UAM land infrastructure categorization and have the capacity to accommodate at least four eVTOL vehicles in parking spaces. In order to simplify the naming system in this paper, we will mention Vertistops and Vertiports as Verti-X.

The UAM planning research project aims to have a holistic and systematic design of Verti-X, taking into account the different requirements. Therefore, the following important aspects are to be investigated and researched:

- Airspace management such as flight corridors, height restrictions in the surrounding area, and flight safety regulations.

- $\quad$ Aircraft operation management, passenger comfort, security and emergency management, microclimatic conditions, emission control, visuality, and public acceptance.

- Elevated Verti-X should also consider the building physics regarding shocks and vibrations, space requirements, integration of infrastructure of charging, parking, and maintenance. Additionally, noise protection in the building, safety regulations, such as fire protection and personal rescue, should be considered as well.

- Passenger moving chains of routes, comfort, safety, acceptance, access, and information, the attractiveness of Verti-X as a location.

- Urban space with location criteria as a view of urban development and traffic vision.

In this respect, all aspects of the specifications must be taken into account by the legislator, the DIN (German Institute for Standardization), the facilities, the buildings, and the areas required. The regulatory and infrastructural problems of the cities are, however, only partially addressed, although the inclusion of cities and municipalities has been stated. Looking at the current situation in the cities that actively support the UAM problem, it can be seen that a very interdisciplinary, multidimensional list of requirements is facing them. Different criteria have to be defined both qualitatively and quantitatively for the provision of Verti-X for flight taxis. Therefore, the following research aspects arise for this paper:

- $\quad$ existed methods to enable the holistic and systematic approach of the Verti-X design;

- Verti-X location to be planned with corresponding requirements;

- $\quad$ existing roof surfaces of buildings or parking garages to be used with qualitative and quantitative requirements;

- $\quad$ standards, regulations, and constructional requirements must be used as a basis - if changes are needed;

- $\quad$ essential parameters and requirements such as noise and emissions to be incorporated and taken into account in planning.

This paper focuses on how agile concepts could help to prepare Verti-X in an ongoing phase. We are laying the foundation for Verti-X growth, particularly in urban environments. We take into account the interactive monitoring of Verti-X and its network, the function of Verti-X as part of integrated mobility principles during take-off and landing, and air traffic management systems (landing site communication). We will delve deeper into these research questions in the following section and answer them based on our research findings. The first section will provide a summary of the context and meanings of UAM and Verti-X as a reminder of the paper. Section two will present the current state of the Verti-X architecture. In section three, this paper will show how, with the emphasis of Verti-X, the MAGIC approach has been applied to design and plan the infrastructure component of UAM. The last part will summarize the outcomes. 


\section{STATE OF THE ART}

In this section, we would like to demonstrate the related work regarding the design of Verti-X:

- The Verti-X standard description: to answer the question - what to consider.

- The Verti-X location criteria: to answer the question - where to locate.

- The Verti-X characteristics with specific application details: to answer the question - how to apply.

\subsection{Related Research for Requirement Analysis}

In recent years, many researchers and eVTOL producers (Uber Elevate, 2017) have been studied and discussed about analyze UAM operational constraints (Vascik et al., 2018; Vascik and Hansman, 2017). Less attention has been given to investigate the elements and requirements for a fully functioning urban air mobility system and specifically, the Verti-X. Nevertheless, Verti-X, in this scenario, plays one of the key roles in the UAM system as ground infrastructure. Therefore, a thorough and fundamental requirement analysis of Verti-X should also take UAM system requirement into account as well.

- Infrastructure: restricted space on vertiports allows the overall footprint of vehicles to be reduced. There is a substantial effect of the approved footprint on the feasibility of implementation. In addition, large vehicle footprints lead to reduced efficiency on the Verti-X as less aircraft can be dispatched simultaneously (Ploetner et al., 2020). To allow scaling, the Verti-X must be readily accessible to customers and have sufficient passenger and aircraft throughput.

- Capacity Estimation based on Location: to achieve the maximum societal benefit, operators should place Verti-X in those spots that help reduce congestion - and provide equal access to different types of passengers and cargo. As we have mentioned above, the scale of Verti-X should also be defined based on the location, existed infrastructure such as building conditions, and estimated throughput in the target area. Based on the population density, medium income, office rent price, point of interest such as parking house or large parking lot, major transport node, annual transport cost, job density, extreme commuter, existing helipads location, and existing noise (Fadhil, 2018).

- Take-off/landing Dimension Estimation: Based on the heliport design requirements, the dimensional dimensions of TLOF (Touchdown and Lift-off) pads, exits, staging stands, and taxiways were determined. UAM TOLAs should have requirements of design which are equivalent to those recommended for general aviation. Verti-X high-capacity aircraft were assessed to identify various techniques to coordinate and control aircraft operations among the Verti-X components. (Pukhova et al, 2019).

- Safety: safety problems may cause the restriction of community acceptance, lead directly to the grounding of the vehicle and reduce customer service demand (Vascik and Hansman, 2018). UAM aircraft that need to operate in accordance with the full spectrum of manned and unmanned aircraft with a wide variety of equipment and capabilities currently operating in the airspace. In addition, UAM operations typically occur in metropolitan areas that are densely populated in terms of passenger, unlike traditional aviation, which normally spends the majority of flight time in sparsely populated areas by design (Thipphavong et al., 2018).

- Community Acceptance: Although UAM 's community acceptance can be affected by various factors including privacy, visibility, emissions, protection, and equity, aircraft noise has been the most common externality of aviation leading to community-imposed limits on the scale of the previous helicopter and transport aircraft flights (Vascik and Hansman, 2019; Thipphavong et al., 2018). The service can achieve public approval only if it can be shown to work safely. The registration, boarding, on-trip, and delivery approach can address today's customer service issues. Moreover, in order to save time compared to ground transportation alternatives, these trips should be covered at reasonably high speeds. (2019, Boelens).

- Energy Consumption: The demand for electricity will be increased by eVTOL aircraft, which will help to create incentives to shift towards renewable sources of energy, and utility companies will become more responsible for $\mathrm{CO} 2$. On the other hand, aircraft flew over congested areas and crossed every congested area of a town, city, or village, or gathered people in the open air. This implies that eVTOL aircraft are unable to disturb societies, and noise control must be strongly taken into account by aircraft developers (Uber Elevate, 2017).

- $\quad$ Noise Pollution: In addition to vehicle design, eVTOL aircraft operations must take into account and reduce incremental noise emissions and take noise into account in the design and location of 
vertiports and in the construction of new buildings. The current level of noise produced by traffic, both at the surface level and at the airport, is the actual noise level. Noise is one of the main barriers to the introduction of UAM in urban areas (Vascik and Hansman, 2017; Uber Elevate, 2016; Porsche Consulting, 2018).

- Weather Restrictions: it will be important for real-time weather monitoring to notify eVTOL aircraft operations with micro-weather information on wind gusts, fog, lightning, snowfall, and more. Predictive capabilities coupled with monitoring will permit complex and safer route planning. Low visibility, high winds, or poor runway conditions (for instance, due to precipitation) can hinder TOLA operations or require higher performance aircraft and equipment. High temperatures can reduce the effectiveness of aircraft and are especially detrimental to the thermal management of electric aircraft batteries and engines. Finally, icing conditions or convective weather will prevent UAM flights.

The eVTOL producers such as Uber, EHang, and Volocopter have all released their white paper regarding the design of eVTOL, UAM market analysis, and Verti-X infrastructure analysis. At the metropolitan level, point-to-point air links may help resolve the shortage or pollution of surface transport, lighten and supplement logistic chains, and reduce time and recurring maintenance costs.

\subsection{Related Criteria and Regulations}

Currently, there are so far no Verti-X-specific standards, and the industry remains unaligned on the technical visions and needs for vertiports. Thus, the design criteria of Verti-X are established and adapted to the existed criteria of helipad and heliport (Amoroso et al., 2012). There are three critical areas of a helipad, namely TLOF, Final Approach and Take-off (FATO), and Safety Area (SA). TLOF is a circle in the heliport center, acting as a pad for the helicopter to be landed. TLOF is also colocated with FATO, which provides a shielding area when the aircraft is entering or departing the heliport in a glideslope. SA is a circle located in the outermost part of these three key areas of the helipad. The purpose is to include an external buffer. Both TLOF and FATO are located within SA (FAA, 1991). Furthermore, other areas, including the passenger waiting area, parking area, as well as access path, should also be considered.

Therefore, the relevant quantitative and qualitative parameters could be to be determined based on the following regulations:

- The determination of vertipad layout in accordance with the current ICAO SARPs (Standards and Recommended Practices) is based on several factors, notably the aircraft. In particular, the average total size (D) of the aircraft and the efficiency level of aircraft operations. Manuals to ICAO Annex 14 (2016) of the Convention on the International Civil Aviation Organization contains the basic standards and recommendations (SARPs) for the aerodromes.

- Location criteria are a view of urban development, urban planning effects such as height restrictions in the surrounding area. The analysis of building and infrastructural parameters (loads according to DIN 1991 and DIN 1055 for planning landing areas on buildings and multistory car parks) is necessary when building up the rooftop Verti-X. The vibration, wind current, raw construction, statics and building physics, space requirements, integration of charging infrastructure, and noise protection should all follow the regulations.

- Obstacle limitation surfaces should also be considered according to ICAO Annex 14 while designing the Verti-X. It should be noted that identifying obstacle limitation surfaces, in most cases, a state would need to enact some form of regulation restricting the limits of heights of objects, whether it be natural growth or building structures.

- If simultaneous eVTOL operations are required, appropriate separation distances between FATOs need to be determined, ensuring the flight paths for each FATO. According to ICAO Annex 14 Chapter 4 (2016), It is recommended that heliport, in this case, Verti-X, both surface level and elevated, should have at least two take-off climb and approach surfaces. Multiple takeoff climb and approach surfaces are the most desirable to provide the most options for crews.

- Safety first is the most fundamental and primary consideration in Verti-X design. Safety regulations, such as fire protection and personal rescue, are needed to be considered. The Verti$\mathrm{X}$ must comply with the strictest international safety standards. The ICAO provides the relevant SARPS and related guidance material for the provision of RFF (Rescue and Fire Fighting) facilities in Annex 14 Volume II and the Heliport Manual. In order to provide maximum fire 
protection for aircraft crew, passengers, building occupants, and third parties adjacent to the building is to install the DIFFS (Deck Integrated Fire Fighting Systems) system on the vertipad (DiFelici and Wargo, 2016).

\subsection{Characteristic of Different Verti-X Concepts}

In order to holistically and systematically design a Verti-X, we also need to consider the different locations. In general, there are three types of Verti-X: ground-based, elevated, and over the water. Elevated Verti-X locates on a raised structure on land. This can be raised above the ground, usually at least $75 \mathrm{~cm}$ or more, or on a building or parking structure rooftop. In this paper, we focus on the surface-level Verti-X and rooftop Verti-X. As shown in Table 1, the comparison between Surfacelevel Verti-X and Elevated Verti-X is presented. Surface-level Verti-X has advantages such as cost reduction, no weight limits, less response time, more available space. Elevated Verti-X, on the other hand, has fewer obstructions with more approach pathways, less footprint, better security control, and less eVTOL performance. Another portion of the elevated Verti-X permits customers to be dropped off and access automobile or pedestrian egress points to complete their trip.

Table 1. Comparison between surface-level Verti-X and elevated Verti-X (ICAO Annex 14, Volume II - Heliports, 2016)

\begin{tabular}{|c|c|}
\hline Surface-level Verti-X & Elevated Verti-X \\
\hline \hline Significantly reduced cost & $\begin{array}{c}\text { Clear of obstructions with more approach } \\
\text { pathways }\end{array}$ \\
\hline No weight limits & Less footprint \\
\hline Reduced response time & Reduced eVTOL performance \\
\hline Space available for stands and approach lighting & Better security control \\
\hline
\end{tabular}

\subsection{Related Verti-X Design Cases}

Therefore, in order to have a more concrete design, many research studies and eVTOL producers such as Uber, Lilium Plane, Volocopter have devoted themselves to Verti-X design. As shown in Figure 1, numerous Verti-X designs have been demonstrated. Figure 1a shows the vertiport with four parking stands and one pad, as well as one storage space for waiting and maintenance equipment. In a high-rise house, this variant may be positioned along the water embankment, on the ground, or elevated (Fadhil, 2018). Figure $1 \mathrm{~b}$ is proposed by NASA involves building Vertistops within the radius of highway cloverleaf on- and off-ramps, which in many areas are big enough to make for a perfect vertistop space with a generous safety buffer zone and instant car access to the freeway once you're landed (Antcliff, et al. 2016). Figure 1c is proposed by Groupe ADP which includes ground and air operations (UTM), energy and maintenance facilities, and even the future passenger route with mobile platen so that once the eVTOL is in place, various functions can be completed by moving the platen: landing, passenger disembarkation, maintenance, passenger embarkation, take-off (Bernie, 2019).

Figure $1 \mathrm{~d}$ is proposed by a UK design consultancy firm called 'small' (Six Miles Across London Ltd.). The vertiport has a modular design that enables its exterior to be equipped with lounge and seating areas, storage areas for repair and spare batteries, and even small dining and retail facilities. The idea of the vertiport essentially provides a small-scale airport (SMALL. 2018). Figure 1e is Uber's depiction of an artist to demonstrate many of the future features of vertiport and help imagine the form of infrastructure planned as part of a ridesharing network for use with on-demand VTOLs (Uber Elevate, 2017). In this example, according to FAA Aircraft Circular 150/5390-2C (2012), the top of an eight-story downtown parking garage was converted to a vertiport capable of supporting 12 VTOLs. Lilium, which has three common ingredients, is suggested in Figure 1f: a take-off area, parking stands, and a terminal. Additionally, the Verti-X also needs high-power charging equipment, air traffic control technology for high throughput operations, and digital check-in tools (Lilium Jet. 2019). 


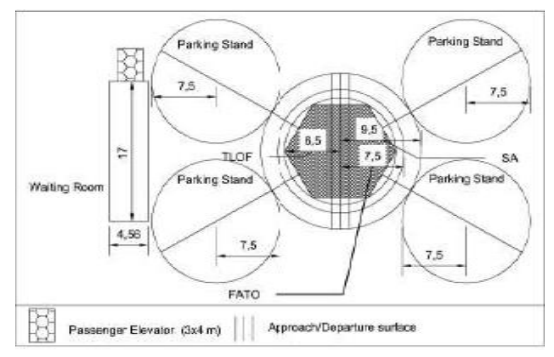

(a)

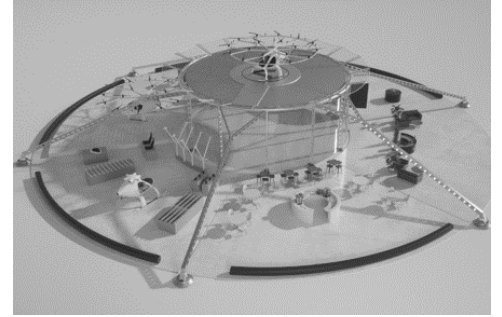

(d)

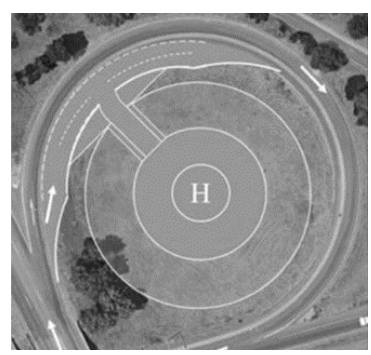

(b)

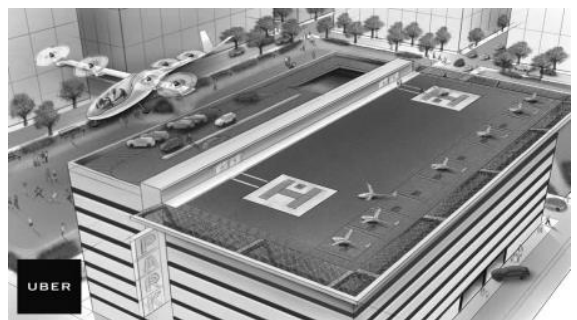

(e)

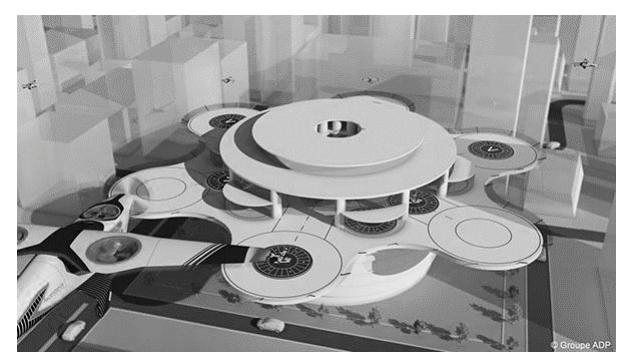

(c)

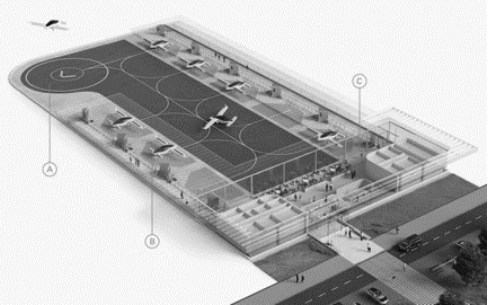

(f)

Figure 1. Related Verti-X design: a) vertiport (Fadhil, 2018) b) highway vertistop (Antcliff et al. 2016) c) Groupe ADP (Bernie, 2019) d) Voloport (SMALL. 2018) e) Uber (Uber Elevate, 2017) f) Lilium Vertiport (Lilium Jet. 2019)

\section{IMPLEMENTATION OF MAGIC APPROACH FOR VERTI-X}

MAGIC approach derived from the shape of the DevOps infinity loop model and consists of 6 levels: system goal and requirement level, system functional level, systems architecture and logical level, system behavior analysis and verification and validation, system production and additive manufacturing and test, system usage and service level. We will explain each level in detail in the following parts, as well as the implementation of the MAGIC approach. (Salehi and Wang, 2019). Compare to traditional systems engineering or model-based system engineering approach, MAGIC approach has several advantages including 1 ) the requirements are continuously adopted to every stage of system development from first product ideas up to manufacturing and product services/usages for a better traceability 2) an agile working and communication are implemented between every state of the MAGIC approach. This ensures to have a close connection during system development phases. 3) the whole spirit of agile is reflected as the iterative and incremental design and development.

- Based on the state of art literature survey mentioned in section two, the main research focus of this paper is clear as following:

- $\quad$ Apply the MAGIC approach to the holistic and systematic design of Verti-X.

- Determine the key components of the Verti-X design with thorough requirement analysis.

- Follow the listed criteria and regulations to design a valid and verified Verti-X which fulfills all the requirements.

- Therefore, in this section, we will adopt the MAGIC approach for the development of Verti-X. As shown in figure 2, adapted MAGIC approach consists of 6 phases which are 1) Verti-X requirements, 2) Verti-X functions, 3) Verti-X architecture, 4) Verti-X Validation/ Implementation, 5) Verti-X Testing and 6) Verti-X Usage/Application. 


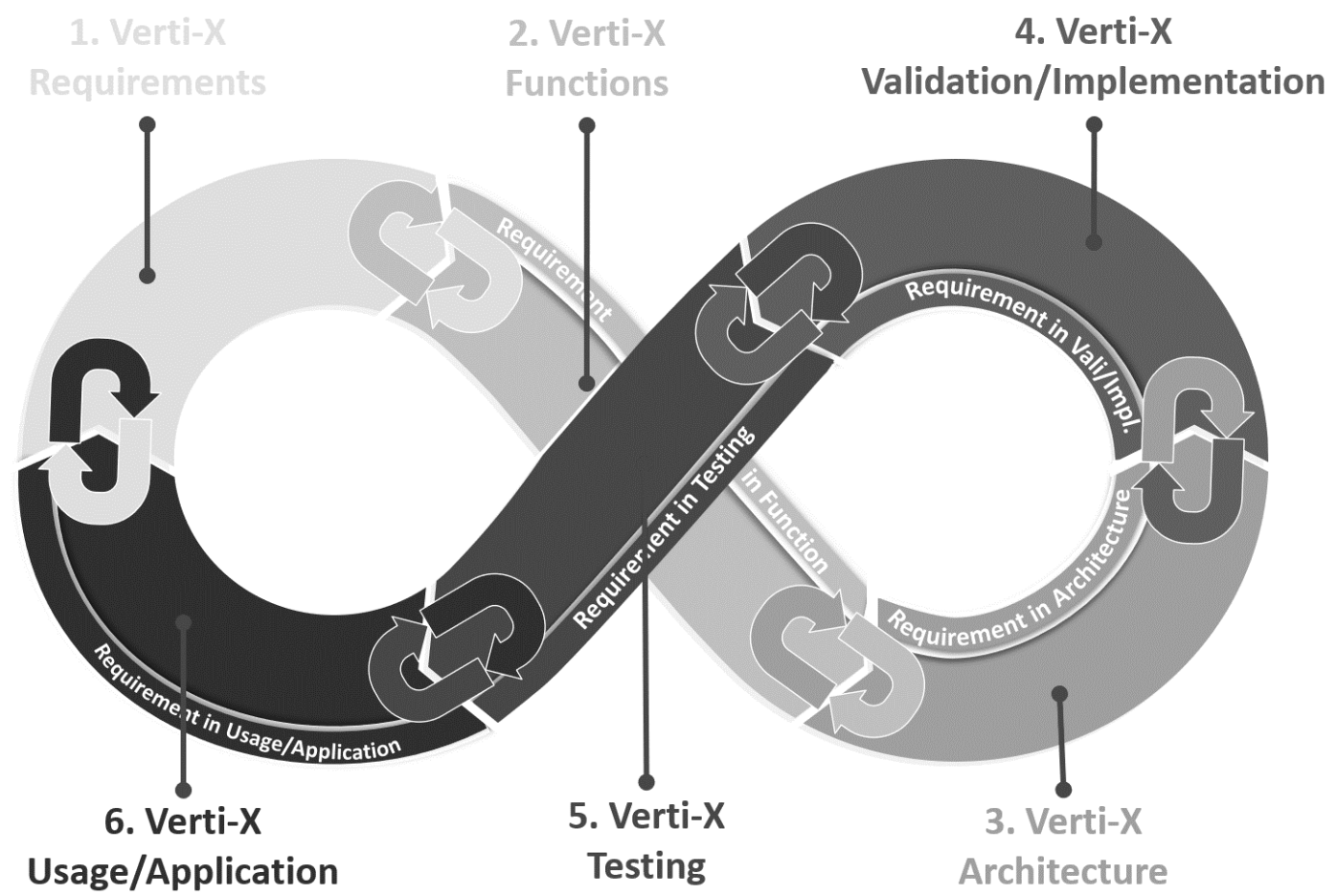

Figure 2: Adoption of the Munich agile concept for development of Verti-X

\subsection{The Verti-X System Requirement Level}

System Requirement level contains all the customers' needs and wishes related to the product and its requirements (Salehi et al., 2011). The challenge during the first stages of product development is to understand and translate the costumers' needs into technical requirements. The system requirement will go through the entire phase of the MAGIC approach to examine the system development stages, and each requirement should be able to trace back (Salehi et al., 2019). In order to have a clear view of the requirement of the development of Verti-X, we will discuss it in 2 aspects, as shown in figure 3 regarding eVTOL requirements and location criteria. The eVTOL aircraft requirements mainly consider the aircraft operation process, passenger's journey, and staff working environment. On the other hand, location criteria involve the infrastructure, accessibility based on the connected transportation node, as well as safety considerations. In the following part, we will discuss it in detail.

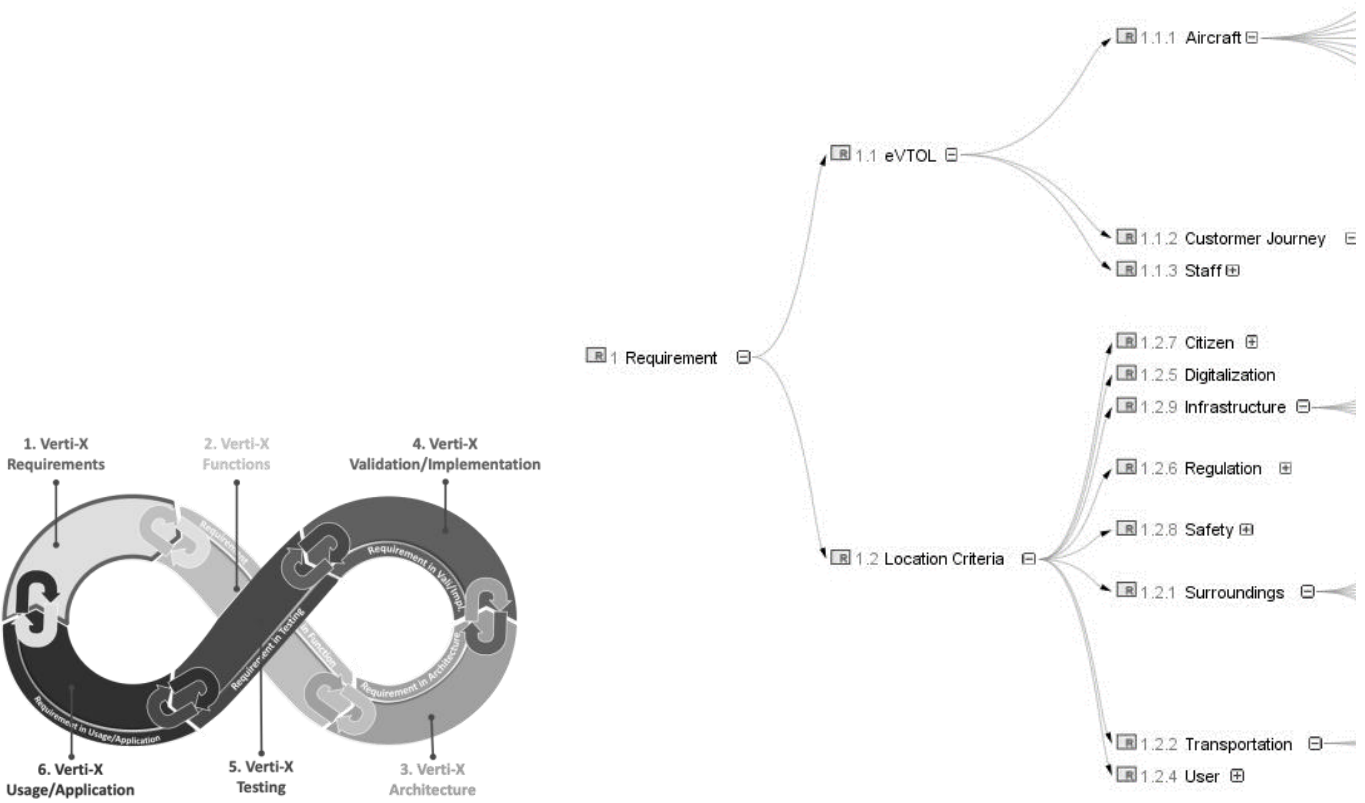

Figure 3. Overview of Verti-X requirements 


\section{Aircraft}

The FATO vertipad shall, in accordance with existing regulations, allow aircraft with the driving dimension D to land. In addition, the vertipad must provide the FATO with a minimum of two horizontally spaced approach directions according to the relevant regulations. Where space permits, a second FATO shall be provided for the vertipad to ensure the simultaneous handling of several aircraft and to enable pilot-controlled parking and pushback operations. In all probable landing situations, the vertipad will assist aircraft with MTOW and have a completely enclosed parking bay devoted to light maintenance and repair operations. The charging infrastructure shall be provided for every parking position and prevent passengers from interfering with the charging infrastructure.

\section{Passenger Journey}

One of the key designs of Verti-X regards to passenger comfort. It expects passenger facilities, including pre-flight monitors, airline lounges, and boarding procedures. Therefore, the Verti-X layout shall allow quick passage between the Verti-X entrance and aircraft parking stands. Parking positions shall enable passengers to get to the aircraft without direct exposure to rain, snow, or other precipitation. The Verti-X location and layout shall enable passengers to reach relevant linked means of transportation quickly and comfortably. Moreover, the Verti-X layout shall offer enough personal space for customers at any given time during regular operation and provide the capacity to accommodate the maximum amount of passengers given the calculated maximum throughput and means to restrict access to unauthorized people.

\section{Infrastructure}

The development of the required eVTOL infrastructure of a city requires a data-driven approach. The biggest operational obstacle to using an eVTOL fleet in cities is the lack of sufficient locations for the placement of landing sites. Even if eVTOLs were approved for flight today, the cities simply do not have the necessary take-off and landing areas for the vehicles to be used on a fleet scale. A small number of cities already have several helipads and could have sufficient capacity to provide a limited eVTOL service, provided that they are in the right locations, are easily accessible from the road level, and have space for additional charging stations. However, the infrastructure needs to be extended if eVTOLs are to realize their full potential. However, because of space availability and expense, the build-out of Verti-X presents a major challenge. Public-private partnerships can help resolve cost barriers, along with the use of a variety of cost-sharing and revenue-sharing models.

\section{Transportation}

The UAV should be able to cover the most popular high-traffic routes in major cities, such as the airport to the city center route. The eVTOL aircraft would be better than operating a vehicle on a perpassenger basis. Because the public may be concerned not just with time spent on city flying traffic, but door-to-door time, there may not be a lot of people willing to try this new thing if the docking point is inconvenient either. Commonly, the vertiport should have an easy connection to public transport such as train stations, bus stations, tram stations, subway stations, parking lot, garage, etc. (Ballin et al., 2002).

\section{Safety}

Passengers shall only be permitted to access the vertiport with valid permission to travel and shall not be permitted to carry prohibited objects to secured areas, to travel and to board non-compliant baggage (size, weight) aircraft. The parking stands are dimensioned to ensure that there is no risk to passengers or employees when travelling around the aircraft when optimizing the efficiency of the vertiport. Reasonable access controls are provided by Verti-X for personnel and protection applies to workers, not just passengers.

\subsection{The Verti-X System Functional Level}

System Functional level is a method for understanding the total product and defining the structure and behaviour of a system (Salehi et al., 2009). A system is structurally represented by the functional or structural relationships between the individual components or sub-functions concerning the technical requirements. Once the functional structure is illustrated, the technical system architecture is created considering the functional structure. The functional design resulting from system requirement and functional analysis should be conducted from the perspective of the Verti-X design of interest. Functional analysis focuses on the intended system behavior necessary to support a concept of operations. 
As shown in figure 4, according to requirement, we start the system decomposition with the functions. A Verti-X should have functions such as enabling charging unit, planning work area for workers, enabling fully fitted vertipad, planning repair bay, designing a waiting lounge, ensuring parking / charging zone, providing boarding / de-boarding route, enabling emergency exit, allowing the requirement to be met by digital check-in / check-out. A functioning Verti-X system also requires a high-speed communications network that accounts for an urban environment's impact on availability and resiliency. An efficient, seamless flow between land and air infrastructure is generated from the independent check-in phase with automated ID verification, to flight boarding and the transition to other urban mobility concepts-for an instant flight experience. Additionally, the boarding experience is important, meaning that operators should provide visual/directional cues and signage to make using eVTOLs an enjoyable part of a passenger's overall journey. Given that many Verti-X will likely be on building rooftops, which might have multiple landing platforms, guiding commuters to the right take-off locations and vehicles.

The device sub-functions point towards the physical configuration of the eventual sub-systems. For example, the charging device's nature is to pass energy to a portable charger and a fixed charger. The waiting lounge should have waiting facilities complete with food and beverage supplies. In addition, parallel behavior must also be considered. The parking and charging zone, for example, should not only allow pilot-control parking, but should also allow pushback operations and the simultaneous handling of multiple parking spaces. In addition, Verti-X must be configured to meet fleet service criteria in order to ensure that during an unplanned interruption, continuity of operations could be maintained.

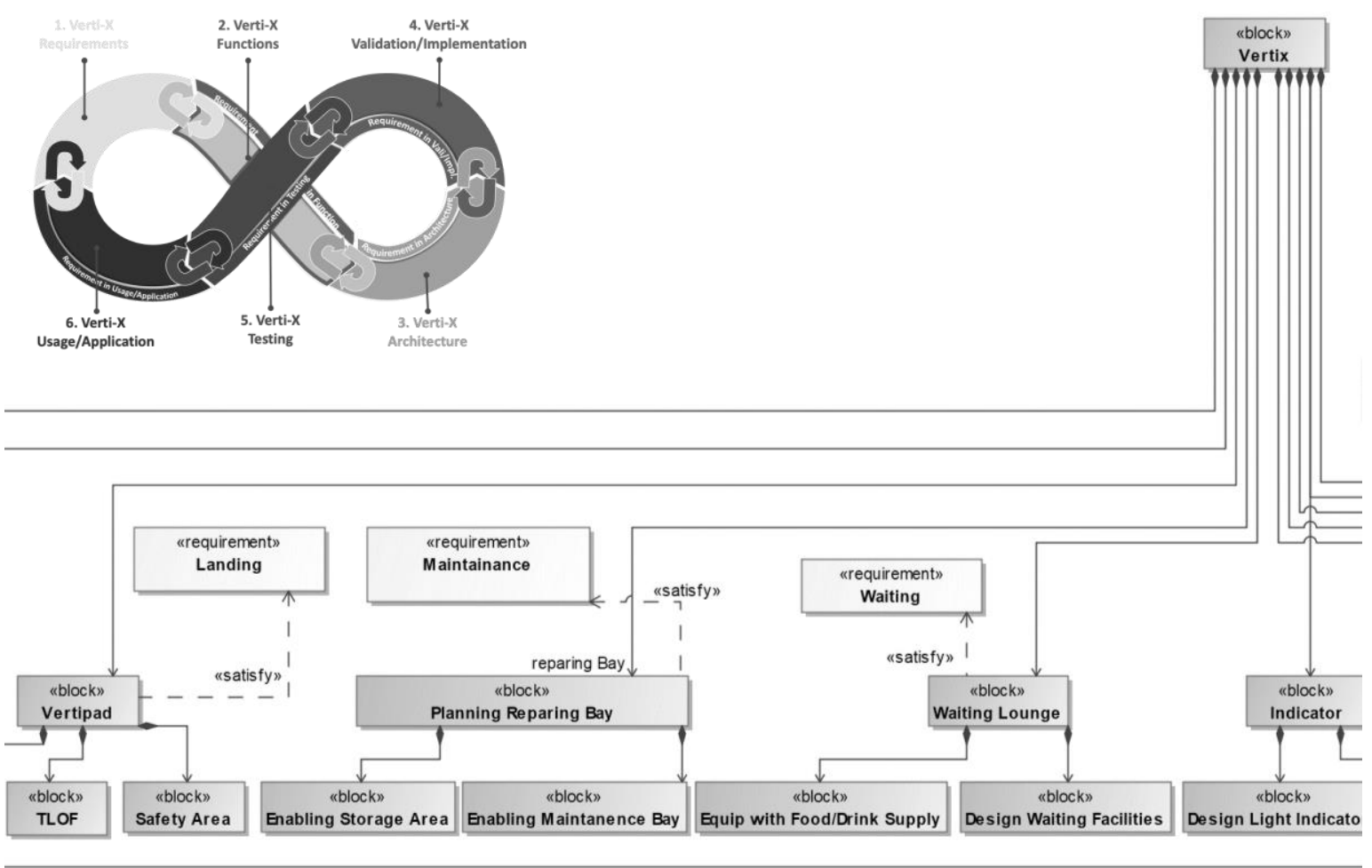

Figure 4. Overview of Verti-X functions

\subsection{The Verti-X System Architecture Level}

This level includes components that have logical ports with the logical behaviour of a system. The technical system architecture of the MAGIC approach describes a rough geometry, components design, and concepts. It defines "how" the functional requirement will be achieved - and there can be multiple ways to accomplish the requirements (Salehi et al., 2018). This stage explains rough geometry, design of components and principles, models of kinematics. The Verti-X concept is basically a small-scale airport. The modular design enables Verti-X to adapt flexibly to various locations and to be incorporated into roofs, parking lots or other urban structures. It also enables us, depending on the venue, the passenger demand and available space, to customize our standardized layouts. The Verti-X module consists of a set of functional modules that are configured for regulatory 
enforcement and efficient operations. Additionally, keeping the design lean and modular will allow the Verti-X network to grow rapidly to deliver high-speed connectivity in a fraction of the time. Therefore, whether a Verti-X is designed to support small or large scale of throughput operations, it should have three common ingredients: an eVTOL operation area, a parking zone and a terminal as shown in figure 5 .

- The eVTOL operation area could be on the surface level or elevated. It includes the vertipad, emergency exit, boarding/de-boarding path, and parking zone with high-power charging equipment. Elements need to be designed for vertipad consist of FATO area, TLOF area as a focal point for operations and will be surrounded by a safety SA area, and light or text indicators. The design of vertipad is driven by established guidance for heliports with approach slopes and obstacle clearance guidelines. On the ground, the eVTOL will travel under its own power using electric motors along defined taxiways to and from the parking stands.

- The parking zone should allow the storage areas for repair, replacement of batteries, and maintenance of eVTOL. The parking zone dimension are sized to ensure there is no danger to passengers or colleagues while moving around the aircraft, while also maximizing the efficiency of the Verti-X.

- The design of a terminal focuses on delivering a seamless and frictionless experience for passengers. Therefore, it should focus on reducing processing and waiting to a minimum. Besides, the terminal consists of digital check-in/check-out, supports even small dining and retail areas, staff working zone, as well as signs. Moreover, ground communication base with air traffic control technology for high throughput operations is also significant. The monitoring and regulation of eVTOL flights includes a comprehensive traffic management system to provide overall operations with centralized control. Scheduling and sequencing, guiding takeoffs and landings and maintaining the proper distance between eVTOLs in the air should be the responsibility of a traffic management system.

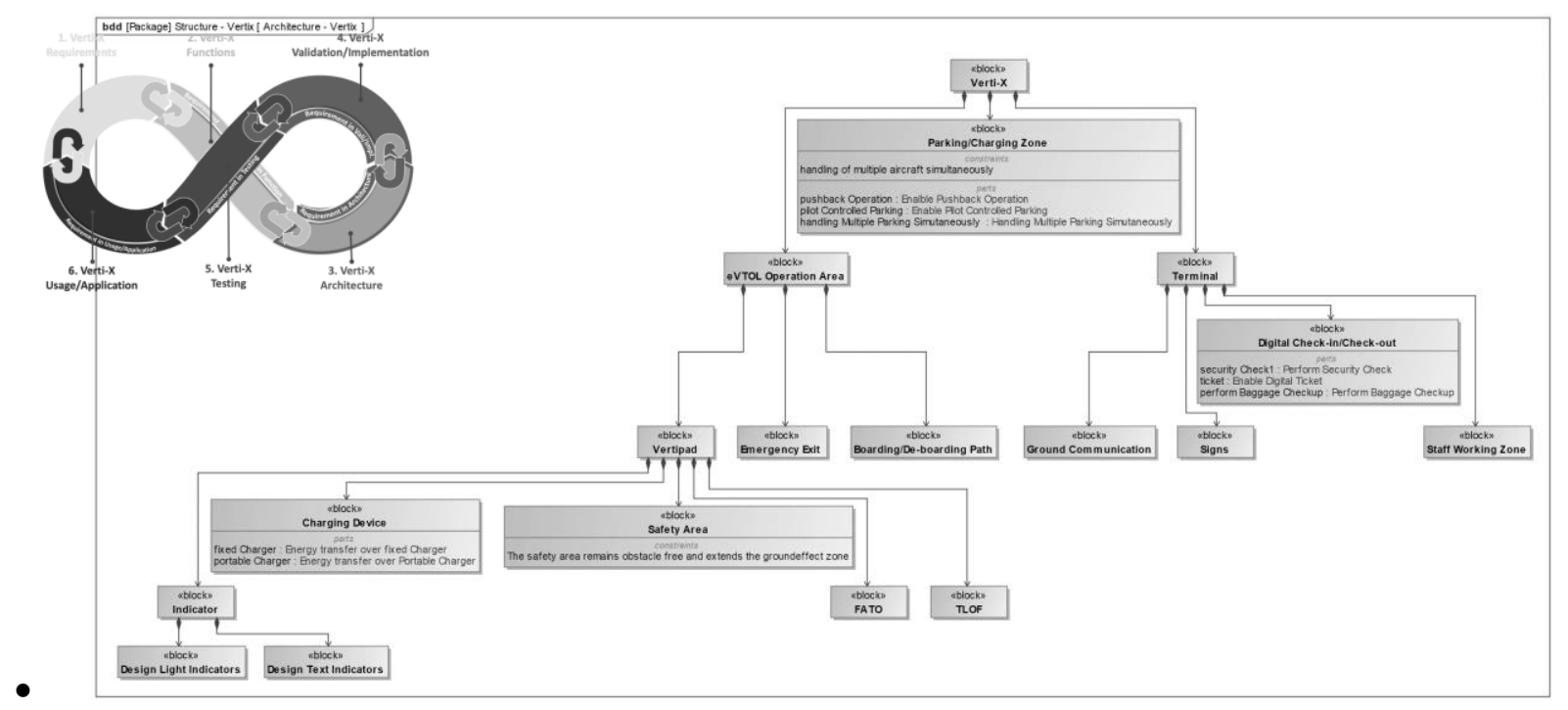

Figure 5. Overview of Verti- $X$ architecture

\subsection{The Verti-X System Validation \& Implementation Level}

System Validation is to be understood as meaning testing whether the product is suitable for its intended purpose or achieves the desired value. Validation comprises, for example, checking whether the description of an algorithm coincides with the problem to be solved. It generally does not have to be carried out formally (ISO/IEC TS 24748-1, 2016). System Validation is a collection of actions used to verify the compliance with the intent and functions of any element (a system unit, a system, a document, a service, a mission, a system requirement, etc.). As shown in figure 6, we have generated a satisfy requirement matrix to verify whether the designed component fulfil the requirement. In general, satisfy requirement matrix consists of 1) row - a requirement element that can be the client element of the satisfy dependency; 2) column - a system architecture element that can be the supplier element of the satisfy dependency. In this case, based on the number of satisfactions, we could clear trace if our design of Verti-X has fulfilled each requirement which has been defined in the beginning. 

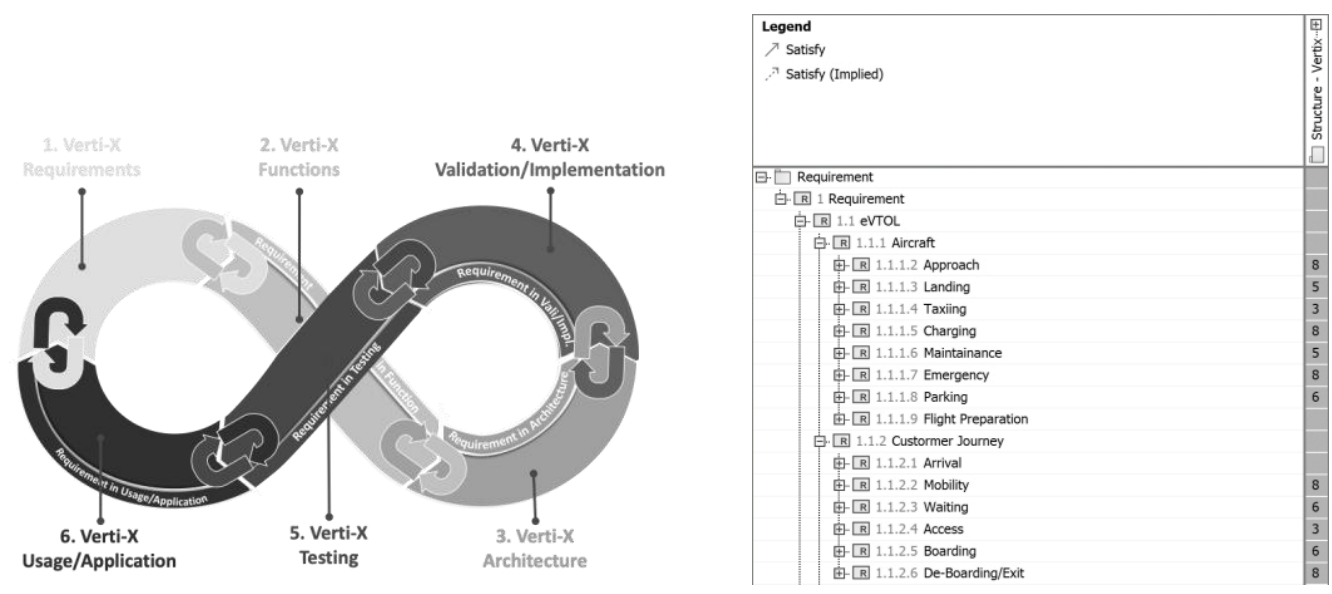

Figure 6. Overview of Verti- $X$ validation diagram

\subsection{The Verti-X System Testing Level}

In order to achieve automation and data exchange with manufacturing technologies, in this stage, the goal of system testing is to discover where the Verti-X does not fit or contradict the description of the system by contrasting it with the description of system requirements. In addition, practical testing of ground operations and facilities is also required, including swapping and recharging of batteries, repairs, and safety.
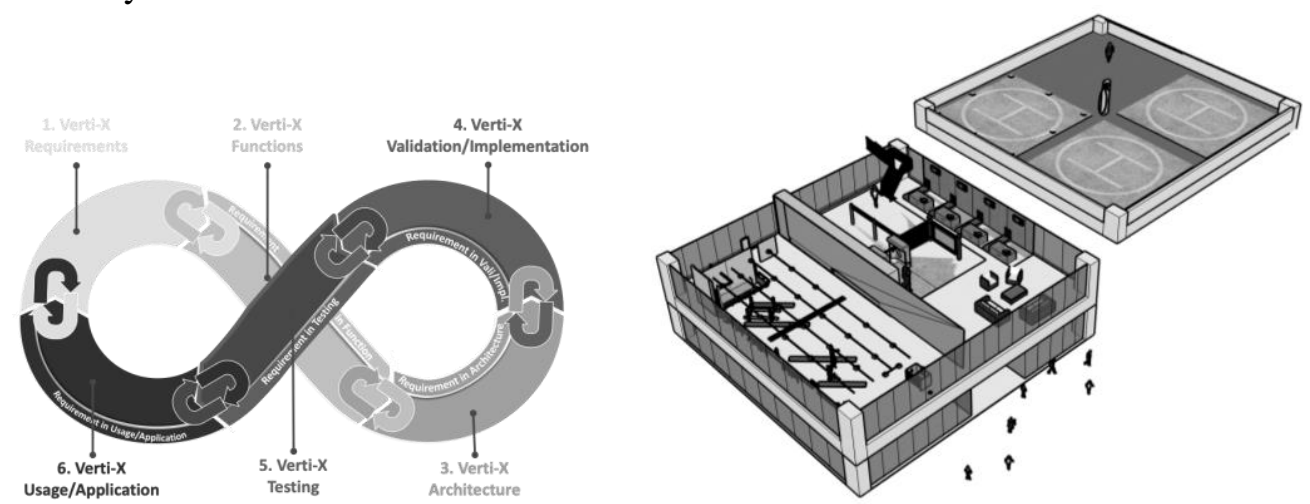

Figure 7. Overview of Verti-X testing diagram

\subsection{The Verti-X System Usage \& Application Level}

In this level, the focus is on the impact of usage on different application scenarios with customer satisfaction. Anyone flying over the city in an eVTOL would like to experience the excitement and joy that comes with such a flight. In order to achieve that, the Verti-X and transition paths shall provide sanitary facilities, trash bins, refreshments, and other amenities. The Verti-X shall enable efficient terminal operations, offer means to visual monitoring of the lounge, terminal, and apron area. The experience will be enhanced by features that predict fees and arrival times, help travelers navigate in and around vertiplaces and provide incoming information to vertiport operations, such as notifying a vertiplace of the pending arrival of a passenger and planning to use facial recognition to transfer users through protection.

As shown in Figure 8, four categories of actors may be involved in an application case: aircraft mechanics, ground workers, passengers as well as pilots. In this instance, however, we assume that the eVTOL aircraft was operating in unmanned autopilot mode. Therefore, in order to take the eVTOL aircraft, passengers need to conduct a sequence of activities such as security checks, automated checkin, and boarding. Activities such as security checks often include the ground personnel. Furthermore, the aircraft engineer is responsible for routine check-ups and repair of eVTOL aircraft. 


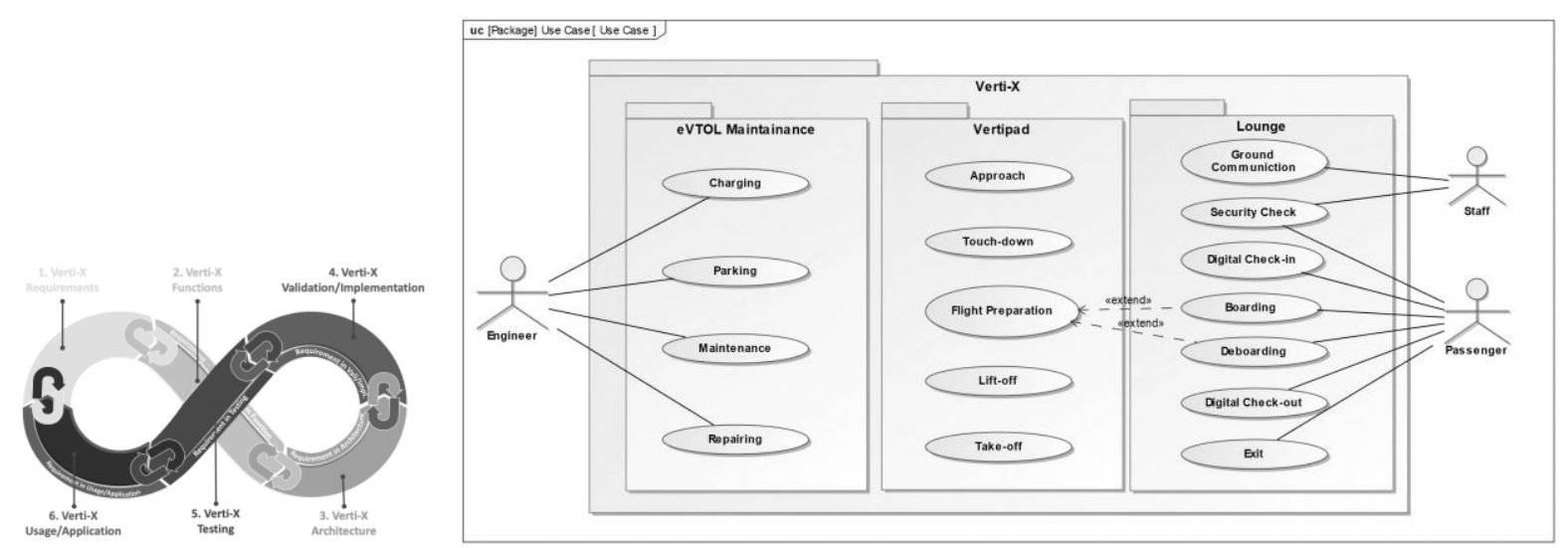

Figure 8. Overview of Verti-X usage and application diagram

\section{CONLUSION AND DISCUSSION}

Urban air mobility in urban and suburban areas is planned to improve the transport of people and cargo. But eVTOL aircraft can not reduce transportation time and emissions and improve performance and public safety without a robust UAM system. Indeed, infrastructure is probably the biggest challenge to be addressed in creating scaled implementation of this new form of UAM. The costs associated with land / space acquisition and development require strong public-private cooperation. In this paper, based on Munich Agile Concept's step-by-step guidance, a detailed requirement review is performed to demonstrate a clear view of the Verti-X implementation. The development of a holistic and systematic Verti-X considers the aircraft operation process, passenger's journey, staff working environment, location criteria regarding the infrastructure, accessibility based on the connected transportation node, safety considerations as well as corresponding regulations. We addressed the qualitative and quantitative analyzes of specifications in various ways based on the proposed research questions. Besides, noise and pollution standards are also briefly listed. This paper explores how agile concepts can help Verti-X plan for an ongoing process. Particularly in urban environments, we generate the basis for Verti-X growth. As part of advanced usability concepts, we take Verti-X and their collaborative network control, Verti-X operation during take-off and landing, and air traffic management systems into account. We could see in the near future, the eVTOLs are a potential mass public transit style that can be ecological and efficient in a specific way. In addition, public acceptance of UAM is likely to be more difficult than mainstream opinion requiring. Therefore, for further research, we plan to conduct the simulation and visualization of a Verti-X to test the feasibility of UAM planning.

\section{REFERENCES}

Amoroso, S., Migliore, M., Catalano, M., Castelluccio, F. (2012), "Vertical take-off and landing air transport to provide tourist mobility". Journal of Air Transport Management, Vol. 24, pp. 49-53. https://https://dx.doi.org/10.1016/j.jairtraman.2012.06.006

Antcliff, K., Goodrich, K., Moore, M. (2016), “NASA Silicon Valley Urban VTOL Air-Taxi Study”. NASA Langley Research Center

Anderson, K., Blanchard, S., Cheah, D., Koling, A., Levitt, D. (2015). "City of Oakland Mobility Hub Suitability Analysis Technical Report”. (December).

Ballin, M. G., Hoekstra, J. M., Wing, D. J., Lohr, G. W. (2002) "NASA Langley and NLR Research of Distributed Air/Ground Traffic Management". AIAA's Aircraft Technology, Integration, and Operations (ATIO). AIAA. https://doi.org/10.2514/6.2002-5826

Bernie, P. (2019), Groupe ADP unveils its vertiport model airport. Retrieved from http://www.aerobernie.com/2019/06/groupe-adp-unveils-its-vertiport-model-vtol.html

Boelens, J. H. (2019), "Pioneering the Urban Air Taxi Revolution 1.0. Volocopter: Bruchsal, Germany", Retrieved from https://press.volocopter.com/images/pdf/Volocopter-WhitePaper-1-0.pdf

DiFelici, J., Wargo, C. (2016). "UAS Safety Planning and Contigency Assessment and Advisory Research". Integrated Communications Navigation and Surveillance (ICNS) IEEE. https://doi.org/10.1109/ICNSURV.2016.7486388

European Aviation Safety Agency (EASA). (2018). "Special Condition for VTOL and Means of Compliance". Retrieved from https://www.easa.europa.eu/sites/default/files/dfu/SC-VTOL-01\%20proposed.pdf 
FAA Aircraft Circular 150/5390-2C, (2012) Retrieved from http://www.faa.gov/documentLibrary/media/Advisory_Circular/150_5390_2c.pdf

Fadhil, D. N. (2018). “A GIS-Based Analysis for Selecting Ground Infrastructure Locations for Urban Air Mobility”. Master's Thesis. Technical University of Munich.

Federal Aviation Administration, (1991) “AC 150/5390-3: Vertiport Design,” Department of Transportation, Washington, DC

ICAO Annex 14, Volume II. (2016). Retrieved from https://www.bazl.admin.ch/dam/bazl/de/dokumente/Fachleute/Regulationen_und_Grundlagen/icaoannex/icao_annex_14_aerodromesvolumeiiheliports.pdf.download.pdf/icao_annex_14_aerodromesvolumeii-heliports.pdf

ISO 9000 (2015), “Quality management systems - fundamentals and vocabulary”, 4th edition, ISO Copyright office, Geneva.

ISO/IEC TS 24748-1 (2016), "Systems and software engineering -- Life cycle management -- Part 1: Guidelines for life cycle management ISO Copyright office", Geneva.

Lilium Jet. (2019) Lilium reveals new air taxi as it celebrates maiden flight. Retrieved from https://lilium.com/technology

Ploetner, K. O., Al Haddad, C., Antoniou, C., Frank, F., Fu, M., Kabel, S., et al. (2020). "Long-term application potential of urban air mobility complementing public transport: An upper Bavaria example". CEAS Aeronautical Journal, pp. 1-17.

Porsche Consulting. (2018). "The Future of Vertical Mobility”. Retrieved from https://www.porscheconsulting.com/en/press/knowledge/the-future-of-vertical-mobility/

Pukhova, A., Llorca, C., Moreno, A. T., Zhang, Q., Moeckel, R. (2019). "Urban air mobility: another disruptive technology or just an insignificant addition”. In European Transport Conference. Dublin, Ireland.

Salehi V., Mcmahon C. (2009), "Action Research into the use of parametric associative CAD systems in an industrial context”. International Conference on Engineering Design ICED’09, Stanford University, Stanford, CA, USA, 24 - 27 August 2009.

Salehi V., Mcmahon C. (2009), "Development of a Generic Integrated Approach for Parametric Associative CAD Systems”. International Conference on Engineering Design ICED’09, Stanford University, Stanford, CA, USA, pp. 24 - 27 August 2009.

Salehi V., Mcmahon C. (2011), "Development and Application of an Integrated Approach for Parametric Associative CAD Design in an Industrial Context”, Computer Aided Design and application, Vol. 8, pp. 225-236.

Salehi V., Mcmahon C. (2011), "Development of an evaluation framework for implementation of parametric associative methods in an industrial context”. International Conference on Engineering Design ICED'11, DTU University, Copenhagen, Denmark, August 2011.

Salehi, V., Florian, G., Taha, J. (2018), "Implementation of Systems Modelling Language (SysML) in Consideration of the CONSENS Approach". Proceedings of the DESIGN 2018 15th International Design Conference, pp. 2987-2998. https://doi.org/10.21278/idc.2018.0146

Salehi, V., Wang, S. (2017), "Using point cloud technology for process simulation in the context of digital factory based on a systems engineering integrated approach". Proceedings of the 21 st International Conference on Engineering Design (ICED17), Vol. 3: Product, Services and Systems Design, Vancouver, Canada, 21.-25.08.2017

Salehi, V., Wang, S. (2019), "Munich Agile MBSE Concept (MAGIC)". Proceedings of the Design Society: International Conference on Engineering Design, Vol. 1 No.1, pp. 3701-3710. https://doi.org/10.1017/dsi.2019.377

Six Miles Across London Ltd. (2018) “UK Architecture Firm Unveils Vertiport Design”. Retrieved from https://transportup.com/headlines-breaking-news/uk-architecture-firm-unveils-vertiport-design/

Thipphavong, D. P., Apaza, R., Barmore, B., Battiste, V., Burian, B., Dao, Q., et al. (2018). "Urban air mobility airspace integration concepts and considerations". In 2018 Aviation Technology, Integration, and Operations Conference. pp. 3676

Uber Elevate: Fast-Forwarding to a Future of On-Demand Urban Air Transportation. (2016). Retrieved from https://www.uber.com/elevate.pdf

Vascik, P. D., Hansman, R. J. (2018). "Scaling constraints for urban air mobility operations: Air traffic control, ground infrastructure, and noise". In 2018 Aviation Technology, Integration, and Operations Conference. pp. 3849.

Vascik, P. D., Hansman, R. J. (2019). "Development of vertiport capacity envelopes and analysis of their sensitivity to topological and operational factors". In AIAA Scitech 2019 Forum. pp. 0526.

Vascik, P.D., Hansman, R.J., (2017). "Constraint Identification in On-Demand Mobility". MIT Int. Cent. Air Transp. 


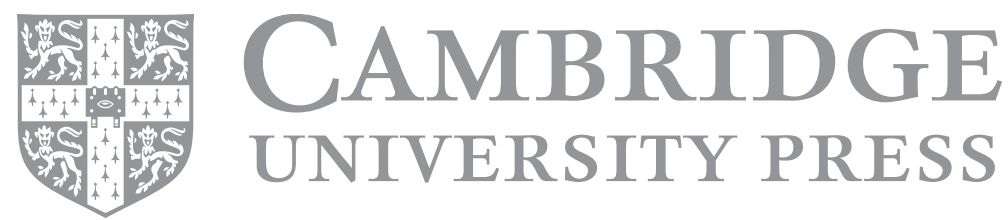

